\title{
1 Spin-enhanced nanodiamond biosensing for ultrasensitive 2 diagnostics
}

3 Benjamin S. Miller ${ }^{1,2 *}$, Leonard Bezinge ${ }^{1}$, Harriet D. Gliddon ${ }^{1}$, Da Huang ${ }^{1}$, Gavin Dold ${ }^{1,3}$, 4 Eleanor R. Gray ${ }^{1}$, Judith Heaney ${ }^{4}$, Peter J. Dobson ${ }^{5}$, Eleni Nastouli ${ }^{6}$, John J. L. Morton ${ }^{1,3}$ \& 5 Rachel A. McKendry ${ }^{1,2 *}$

1. London Centre for Nanotechnology, University College London, 17-19 Gordon Street, London WC1H OAH, United Kingdom.

2. Division of Medicine, University College London, Gower Street London WC1E 6BT, United Kingdom.

3. Department of Electronic \& Electrical Engineering, University College London, London WC1E

7JE, United Kingdom.

4. Advanced Pathogens Diagnostic Unit, UCLH, London NW1 2BU, United Kingdom.

5. The Queens College, University of Oxford, OX1 4AW, United Kingdom.

6. Department of Virology, UCLH, London NW1 2BU, United Kingdom.

The quantum spin properties of nitrogen-vacancy defects in diamond have diverse applications including quantum computing and communications ${ }^{1}$, but nanodiamonds also have attractive properties for in vitro biosensing, including brightness ${ }^{2}$, low $\operatorname{cost}^{3}$, and selective manipulation of their emission ${ }^{4}$. Nanoparticle-based biosensors are vital for early disease detection, however, often lack the required sensitivity. Here we investigated fluorescent nanodiamonds as an ultra-sensitive label for in vitro diagnostics, using a microwave field to modulate emission intensity ${ }^{5}$, and frequency-domain analysis ${ }^{6}$ to separate the signal from background autofluorescence ${ }^{7}$, which typically limits sensitivity. We focused on the common, low-cost lateral flow format as an exemplar, achieving detection limits of $8.2 \times 10^{-19} \mathrm{M}$ for a biotin-avidin model, $10^{5}$-fold more sensitive than gold nanoparticles; and a use-case demonstration of single-copy detection of HIV-1 RNA with a short 10-minute isothermal amplification step, including a pilot using a clinical plasma sample with an extraction step. This ultra-sensitive quantum-diagnostics platform is applicable to numerous diagnostic test formats and diseases with the potential to transform early diagnosis, benefiting patients and populations. 
Fluorescent nanodiamonds (FNDs) containing nitrogen-vacancy (NV) centres (defects with optical transitions within the band gap) have received considerable attention as a spin system for use as a qubit in quantum computing and communication, and for quantum sensing ${ }^{1,4,8,9}$. Such applications stem from the ability of the $\mathrm{NV}^{-}$spin state to be optically initialised and read out, while being manipulated using DC and microwave magnetic fields. FNDs also have attractive fluorescent properties: high quantum yield, non-blinking, no photobleaching, stability, low cytotoxicity ${ }^{2,10}$, available surface groups for biofunctionalisation $^{11}$, and easy mass manufacture, such as from milling of high pressure, high temperature diamond ${ }^{3,12}$. The sensing applications of NV centres ${ }^{4}$ include magnetic field quantification $^{13-15}$, temperature sensing ${ }^{16,17}$, and biological labelling ${ }^{2,18}$, the latter including cellular imaging ${ }^{19}$, drug delivery ${ }^{20}$, and MRI contrast enhancement ${ }^{21}$. A key advantage of negative NV centres $\left(\mathrm{NV}^{-}\right)$is that their fluorescence can be selectively modulated by spin manipulation ${ }^{4}$ (neutral $\mathrm{NV}^{0}$ centres cannot), allowing for signal separation for imaging in high-background environments. This property has been used to improve the contrast for imaging by modulating the fluorescence with microwaves ${ }^{5,22}$, magnetic fields ${ }^{23,24}$, or nearinfrared light ${ }^{25}$. Here, we investigated the use of FNDs for in vitro diagnostics.

Communicable diseases represent an enormous global health challenge, disproportionately affecting poorer populations with limited access to healthcare ${ }^{26}$. At the end of 2015, there were 36.9 million people living with HIV worldwide, of whom 9.4 million (25\%) were unaware of their HIV status ${ }^{27}$. Early diagnosis is crucial for effective treatment and prevention, benefiting patients and populations. For example, UK patients starting antiretroviral therapy for HIV following a late diagnosis saw a reduction in life expectancy of over 12 years compared to those starting treatment with an earlier diagnosis ${ }^{28}$. The earliest marker of HIV is viral RNA, detectable seven days before antigen and 16 days before antibodies $^{29}$. Point-of-care nucleic acid testing, therefore, offers the potential for earlier diagnosis than either existing laboratory-based nucleic acid tests, or point-of-care protein tests. 
Rapid point-of-care tests have transformed access to disease testing in a variety of community settings, including clinics, pharmacies and the home ${ }^{30}$. Among the most common tests worldwide are paper microfluidic lateral flow assays (LFAs), with 276 million sold in 2017 for malaria alone ${ }^{31}$. LFAs satisfy many of the REASSURED criteria ${ }^{32}$ for diagnostics, however, despite widespread use they are still limited by inadequate sensitivity to detect the low levels of biomarkers necessary for early disease detection.

Fluorescent markers can be highly sensitive, but are practically limited by background fluorescence from the sample, substrate, or readout technique. In the case of nitrocellulose

67 substrates used in LFAs, there is a significant background autofluorescence ${ }^{7}$, which inherently limits sensitivity. Various methods have been reported to reduce this effect, such as membrane modification to reduce background fluorescence ${ }^{33}$, exciting in the nearinfrared range and using upconverting nanoparticles ${ }^{34}$, and time-gated detection using longpersistent phosphors ${ }^{35}$ to separate background fluorescence, which has a shorter lifetime.

72 These methods have shown $\sim 10$-fold improvements in sensitivity over gold nanoparticles, 73 limited by relatively low brightness.

Here we show the use of FNDs as a fluorescent label in an LFA format as a

\section{Results and discussion}

An illustration of the use of FNDs in LFAs is shown in Figure 1. FNDs can be used as nanoparticle labels on nitrocellulose strips, undergoing a multiple step binding assay with

85 little user input to bind at the test line in the presence of the target nucleic acids. Once 
immobilised, FND fluorescence can be modulated at a fixed frequency using a microwave

87 field, allowing them to be specifically detected and quantified.

Figure 1: Schematic illustration of the use of FNDs in LFAs. (a) Illustration of the concept of fluorescent nanodiamonds (FNDs) in a lateral flow assay (LFA). The binding of DNA modifications causes FNDs to be immobilised at the test line in a sandwich-format LFA. Inset is the atomic structure of a NV' centre, the origin of FND fluorescence. An omega-shaped stripline resonator applies a microwave field over the LFA, modulating the fluorescence intensity. (b) A schematic showing more detail of the principle. FNDs are immobilised at the test line in a sandwich structure in the presence of dsDNA amplicons. Exciting at 550nm (green) produces fluorescence emission centred at $675 \mathrm{~nm}$ (red), imaged with a camera. An amplitude modulated microwave field, applied by the resonator, selectively modulates the fluorescence of the immobilised FNDs at a set frequency. This allows specific separation of the FND fluorescence from background fluorescence in the frequency domain, to improve the signal-to-noise ratio.

\section{Microwave modulation of fluorescent nanodiamond emission on paper}

An energy level diagram of the $\mathrm{NV}^{-}$centre, the origin of FND fluorescence, is shown in Figure 2a. The triplet ground state is optically driven into an excited triplet state, which then radiatively decays back to the ground states. Throughout the process, the electron spin state $\left(m_{s}=0, \pm 1\right)$ is conserved, however, the $m_{s}= \pm 1$ excited state levels can decay into a metastable 'dark' state with a corresponding reduction in fluorescence ${ }^{4}$. Resonant microwave radiation drives electron spin population from the $m_{s}=0$ to the $m_{s}= \pm 1$ levels, reducing fluorescence intensity. The microwave field was produced by a voltage-controlled oscillator connected to an antenna, capacitively coupled to an omega-shaped stripline resonator that provides a uniform peak field over the measurement area (the area of the resonator).

Characterisation of the FND fluorescence and microwave field response was undertaken on the nitrocellulose paper substrate. To investigate the FND fluorescence intensity with microwave frequency on paper, a wideband resonator was used to perform 
continuous-wave electron spin resonance spectroscopy, shown in Extended Data Figure 1ac. A plot of FND fluorescence over a wide frequency range is shown in Figure $2 b$, showing two peaks at $\Delta E=2.87 \mathrm{GHz}$ and $\Delta E *=1.43 \mathrm{GHz}$, corresponding to the triplet level splitting in the ground and excited states, respectively. Figure $2 c$ shows a narrowband resonator, characterised in Extended Data Figure 1d-f, designed to have a resonant frequency at $2.87 \mathrm{GHz}$ with quality factor $Q=100$, which induced a $\sim 3-6 \%$ reduction in measured fluorescence (Extended Data Figure 1f), varying linearly with the microwave input power in dBm (see Extended Data Figure 1g-h).

FND fluorescence excitation and emission spectra are shown in Figure $2 \mathrm{~d}$. The presence of $\mathrm{NV}^{-}$centres is indicated by presence of the zero-phonon line (ZPL) at $\sim 640 \mathrm{~nm}$. Using an amplitude-modulated microwave field to specifically vary FND fluorescence at a fixed frequency allows for the application of a computational lock-in algorithm ${ }^{6}$ (shown schematically in Extended Data Figure 2a), to selectively extract signals at the reference frequency. This lock-in analysis, shown by Igarashi et al. $^{5}$, separates the periodic FND fluorescence from the non-periodic background fluorescence, caused by nitrocellulose autofluorescence, thus improving sensitivity.

The fluorescence modulation is shown in Figure $2 \mathrm{e} \& \mathrm{f}$. Figure $2 \mathrm{e}$ shows pixel variation over time: the test line, where FNDs are immobilised, has a high variance compared to the background, which does not modulate and has low variance. The time-series is shown in Figure $2 \mathrm{f}$ (top) - a square-wave $4 \mathrm{~Hz}$ amplitude-modulated microwave field modulates the fluorescence intensity. Applying the lock-in algorithm over a small frequency range gave the plot in Figure $2 \mathrm{f}$ (bottom), an absolute sinc function, the Fourier transform of a square pulse. The maximum response is shown when the reference frequency matches the modulation frequency at $4 \mathrm{~Hz}$. The optimisation of modulation frequency, sampling frequency, exposure time and measurement time are shown in Extended Data Figures $2 b-e$. Microwave generation was miniaturised using a voltage-controlled oscillator, amplifier and custom power and timing circuit (65mm x 38mm, Extended Data Figure $2 \mathrm{f}-\mathrm{g}$ ). 
Figure 2: Microwave modulation of FNDs on paper. (a) Energy level diagram of a $\mathrm{NV}^{-}$centre. Splitting in the ground and excited states is labelled as $\Delta E$ and $\Delta E *$, respectively. (b) The variation

142 FND fluorescence under different frequency microwave fields, showing dips at energies $\Delta E$ and $\Delta E *$

143 Some peak splitting is observed around the centre frequency due to a 3G magnetic field generated 144 by the resonator (measured with a Gaussmeter). (c) A microscope image of the omega-shaped 145 stripline resonator used to produce a uniform field at $2.87 \mathrm{GHz}$. (d) Excitation and emission spectra 146 of FNDs. The green shaded area shows the filtered excitation light used. The emission spectrum area 147 is reduced under the application of the microwave field. (e) The pixel variation at the test line (with 148 immobilised FNDs) of an LFA strip under an amplitude-modulated microwave field. (f) The variation 149 of mean fluorescence intensity over time under the application of the same amplitude-modulated 150 field (top). Applying at lock-in algorithm over a range of frequencies gives a sinc function peaking at 151 the modulation frequency (bottom).

\section{Fundamental limits: biotin-avidin model}

Following this fluorescence characterisation and optimisation of the modulation parameters, FNDs were functionalised with biomolecules for incorporation into LFAs. FNDs with a polyglycerol (PG) layer were used, as the hydrophilic layer reduces non-specific binding to the nitrocellulose ${ }^{36}$ (Extended Data Figure 3a), a key limitation of LFA sensitivity.

157 Three sizes of FND-PG (dynamic light scattering shown in Extended Data Figure 3b) were 158 functionalised with antibodies via activation of the PG alcohol groups with disuccinimidyl 159 carbonate $(D S C)^{37}$, as shown in Extended Data Figure 3c. Characterisation by scanning 160 electron microscopy, dynamic light scattering and Fourier transform infrared (FTIR) 161 spectroscopy in Extended Data Figure 3d-i showed successful conjugation, with no 162 significant aggregation upon functionalisation and increases in size consistent with the size 163 of the conjugants ${ }^{38,39}$. The number of active binding sites on the FND surface $(600 \mathrm{~nm}$ 164 diameter) was subsequently quantified using quantitative PCR, as described in Methods and 165 shown in Extended Data Figure 4. The measured value of 4,300 active binding sites per FND 166 is consistent with geometric calculations of the number of antibodies that could bind. 
The fundamental limit of detection (LOD) of FND-based LFAs was investigated using a model biotin-avidin interaction. A serial dilution of BSA-biotin-functionalised FNDs was run on LFA strips with a poly-streptavidin test line, so bound directly (rather than in a sandwich formation), shown schematically in Figure 3a. This high affinity along with the flow rate and 171 high binding capacity of nitrocellulose ensures that the residency time of the FNDs at the 172 test line is much longer than the binding time of biotinylated FNDs to the streptavidin 173 (Extended Data Figure 5a-b). This implies that all the FNDs bind at the test line, making it 174 ideal for benchmarking the best-case sensitivity, and comparing with other nanomaterials. 175 The LODs were quantified for three different particle core diameters: 120, 200 and 600nm.

The resulting LFA test line fluorescence signals were analysed using lock-in analysis, 177 and by conventional intensity analysis (measuring the intensity difference between the test 178 line and background), and compared with gold nanoparticles, commonly used in LFAs ${ }^{40}$. The 179 signal-to-blank ratios (SBRs) were plotted against concentration in Figure 3b for 600nm 180 FNDs. Each dilution series was fitted to a simple linear regression, and the LOD was defined 181 as the intersection of the lower $95 \%$ confidence interval of the linear fit with the upper $95 \%$ 182 confidence interval of the blanks ${ }^{41}$. Figure $3 c$ demonstrates this comparison, with images of 183 the test lines at various concentrations (top). Below (Figure $3 c$ bottom) are time-series of 184 the fluorescence modulation at each FND concentration, showing that signal modulation 185 can be measured well below the concentration at which there is a visible test line.

LODs were 200aM, 46aM, and 820zM for particles of 120, 200 and 600nm diameters, 187 respectively (Extended Data Figure $5 \mathrm{~d}$ ). The larger particles gave the best LODs because the 188 lock-in amplitude scales with the fluorescence modulation intensity, which in turn scales 189 with the number of $\mathrm{NV}^{-}$centres. The number of $\mathrm{NV}^{-}$centres per particle scales with the 190 volume, so the LOD should scale with diameter cubed. Additionally, surface effects reduce 191 the fluorescence of $\mathrm{NV}^{-}$centres close to the surface, so a larger volume to surface ratio 192 should increase fluorescence intensity. 
LODs using 600nm FNDs were $820 z \mathrm{M}$ and $83 \mathrm{aM}$ for lock-in and conventional analysis respectively, yielding a 620-fold improvement in signal-to-background ratio, giving a $100-$

195 fold improvement in the LOD. This increases to an 810-fold improvement in signal-tobackground ratio using $120 \mathrm{~nm}$ FNDs, giving a 380-fold improvement in the LOD.

a $55 \mu \mathrm{L}$ sample. For comparison, the same experiment was performed with $50 \mathrm{~nm}$ gold nanoparticle labels, commonly used in $\mathrm{LFAs}^{40}$ due to their ease-of-functionalisation and strong light absorption. 600nm FNDs were five orders of magnitude more sensitive. Useful gold nanoparticle sizes on LFAs are also limited by the broadening of the plasmonic peak, whilst larger FNDs become brighter. Due to the low numbers of particles detected, the LODs of biological assays are expected to be limited by non-specific binding and equilibrium considerations, rather than the fundamental sensitivity of FNDs.

Figure 3: Characterising the fundamental limit of detection using biotin-avidin binding of FNDs on LFAs. (a) Schematic of the assay - FNDs functionalised with BSA-biotin were run on streptavidinprinted LFA strips, binding directly to the test line. The arrow shows the flow direction. (b) A dilution series of $600 \mathrm{~nm}$ FNDs was measured by both lock-in analysis and conventional intensity analysis, and compared to $50 \mathrm{~nm}$ gold nanoparticles, giving LODs of $820 \mathrm{zM}, 83 \mathrm{aM}$, and $81 \mathrm{fM}$, respectively. Lock-in analysis gave a 100-fold improvement over conventional intensity analysis, and a 98,000-fold improvement over gold nanoparticles. Error bars show standard deviations of three technical replicates $\left(n_{T}=3\right)$, and three measurement replicates $\left(n_{M}=3\right)$ for each sample. (c) An illustration of this comparison, with example images at various concentrations (above), and intensity-time plots (below), showing that a periodic signal is still evident after the test line is no longer visible in the images.

\section{Single-copy detection of HIV-1 RNA with isothermal amplification}

This technology platform was then applied to a proof-of-concept assay, detecting DNA amplicons. The assay is based on a reverse transcriptase-recombinase polymerase amplification (RT-RPA) reaction for the detection of HIV-1 RNA, which is performed with 
modified primers to form a sandwich structure on the nitrocellulose, as shown in Figure 4a. Following assay optimisation, shown in Extended Data Figures 6-8 and described in methods, LFAs were performed with serial dilutions of RT-RPA products using three particle sizes $(120,200$ and $600 \mathrm{~nm})$. The initial aim was to determine the sensitivity of the detection system, rather than the amplification step, so the amplicon concentration used was measured post-amplification. Resulting plots of SBR against amplicon concentration are shown in Figure 4b, and fitted to the Langmuir adsorption isotherm model (Methods Equation 6). The LODs were measured as 9.0, 7.5 and 3.7fM for 120, 200 and 600nm diameter FNDs, respectively. The 3.7fM LOD with 600nm particles corresponds to 2,200 copies/ $\mu \mathrm{L}$, or $1.1 \times 10^{5}$ copies in total (190zmol of DNA).

A model 'amplicon' (described in methods and characterised in Extended Data Figure $9 \mathrm{a}-\mathrm{b})$ was used for a comparison of the $600 \mathrm{~nm}$ particles with $40 \mathrm{~nm}$ gold nanoparticles. The resulting LODs, plotted in Extended Data Figure 9c, show that FNDs give a $\sim 7,500$-fold improvement over $40 \mathrm{~nm}$ gold nanoparticles. The $\sim 13$-fold reduction in improvement over gold nanoparticles compared to the biotin-avidin model is due to non-specific binding. The blanks in the DNA assay have the same FND concentration as the positives, whereas the biotin-avidin assay has 'true blanks' (only running buffer). The resulting small lock-in amplitude in the blanks is $\sim 13$-fold higher than a 'true blank' signal (noise), showing no significant difference from the blanks from the biotin-avidin assay multiplied by this factor of 13 (the two-tailed $t$-test $P$ value $=0.33$ ).

This level of sensitivity from the FND labelling means that a short amplification step before adding to the LFA could lead to single-copy detection, with typical amplification factors for isothermal RPA of $10^{4}$ in $10 \mathrm{~min}^{42}$. This was subsequently demonstrated by performing $10 \mathrm{~min}\left(37^{\circ} \mathrm{C}\right)$ RT-RPA reactions on serial dilutions of HIV-1 transcript RNA, before adding a $6 \mathrm{X}$ running buffer solution to the purified products, and running on LFAs as previously. The resulting SBRs are plotted against RNA input copy number in Figure 4c, showing a LOD of 1copy. Positive results were achieved down to a single RNA copy. Statistical analysis of the lock-in amplitudes (analysis of variance) is shown in Extended Data 
248 Figure $10 a-c$. Due to the 10 -minute amplification time, all concentrations $\geq 1$ copy reach the saturation signal, so a qualitative yes/no result is given. The variation of SBR with amplification time is shown in Extended Data Figure 10d, where single-copy reactions were run for different times. A detectable signal was observed after a 7-minute amplification time. The sensitivity of the FNDs conveys improved LODs in shorter amplification times compared to previous work with RPA using gold nanoparticles ${ }^{43-46}$. In addition, a proof-ofconcept clinical sample (UCLH clinical standard, $4 \times 10^{4}$ copies/ $\mu \mathrm{L}$ ) was successfully detected. This involves the addition of an RNA extraction step, shown in Figure 4d, which would need to be adapted to the point-of-care. RPA has been shown to be relatively robust to complex samples, but this remains a major challenge for the field of nucleic acid testing $^{47}$. The positive clinical standard had a mean SBR of $\sim 19$ compared to the negative plasma control.

In order to demonstrate the suitability of this system for rapid, early disease detection, a small proof-of-concept was performed using a seroconversion panel of thirteen samples taken over six weeks spanning the initial stages of an HIV-1 infection. Extended Data Figure 10e shows that RNA is detected as early as with the RT-PCR gold standard, 264 giving positive results for 6/7 RT-PCR-positive, and 0/6 RT-PCR-negative samples. This is a preliminary study and further optimisation with clinical samples and a larger study is 266 required to precisely ascertain the clinical sensitivity.

Figure 4: Single-copy detection of HIV-1 RNA on LFAs using RT-RPA and FNDs. (a) A schematic of the assay. Digoxigenin and biotin-modified primers were used in a RT-RPA reaction to produce labelled amplicons, which bind to anti-digoxigenin-functionalised FNDs, and streptavidin printed test lines on the LFA strips, forming a sandwich structure in the presence of amplicons. (b) Dilution series of amplicons were run on LFAs for three different particles sizes (120, 200 and 600nm). Serial

272 dilutions were plotted (dots with error bars showing standard deviations, $\mathrm{n}_{T}=3-9, \mathrm{n}_{M}=3$ ), and fitted

273 to the Langmuir adsorption model in Methods Equation 6. Limits of detection for 120, 200 and $274600 \mathrm{~nm}$ diameter FNDs were 9.0, 7.5 and 3.7fM respectively. ${ }^{*}$ marks the lowest concentrations for 275 each particle size that are significantly different from the blanks at the $95 \%$ confidence level, 276 calculated by ANOVA. (c) Serial dilutions of HIV-1 RNA copies were amplified with RT-RPA (10min), 
277 purified, and run on LFAs with 600nm FNDs. The RNA concentration was plotted against signal-to-

278 blank ratio (dots showing the mean, error bars showing standard deviations, and crosses showing

279 individual measurements), with four experimental replicates $\left(n_{E}=4\right)$, and three measurement

280 replicates $\left(n_{M}=3\right)$ for each sample. Single-copy sensitivity was achieved. (d) The system was applied

281 to a proof-of-concept positive clinical sample (UCLH clinical standard), and negative human plasma

282 control, giving a mean SBR of $\sim 19$ and a $P$ value between the negative and positive clinical samples

283 of $8 \times 10^{-13}$ with a $t$ value of -19.3 from an unpaired one-tailed $t$-test.

\section{Conclusions}

Herein, we have shown the use of FNDs as an ultra-sensitive fluorescent label for in vitro diagnostic assays, using microwave-based spin manipulation to increase the signal-tobackground ratio, and therefore sensitivity. The system was demonstrated in an LFA format with two assays. Using a biotin-avidin model, a fundamental LOD of 0.5 particles $/ \mu \mathrm{L}$ was measured, five orders of magnitude more sensitive than gold nanoparticles, with the caveat of increased cost due to the need for a fluorescence reader (see Supplementary Table 1), but the advantage of data capture (compared to visual interpretation). Applying FNDs to a sandwich assay for oligonucleotide detection, single-copy sensitivity was achieved for the detection of RNA with a 10min RT-RPA step, using $600 \mathrm{~nm}$ FNDs. The sensitivity of the FND detection system (LOD of 2,200 copies/ $\mu \mathrm{L}$ with RT-RPA amplicons, measured post amplification) meant a short amplification time is possible whilst achieving higher sensitivity than has been previously demonstrated with other nanomaterials ${ }^{45,48}$, making the test more suitable for point-of-care applications. A comparison with other fluorescence-based amplicon detection on LFAs is shown in Supplementary Table 2. The system was also demonstrated with HIV-1-positive and negative clinical samples with the addition of an RNA extraction step.

There are remaining challenges to translate this exemplar RNA detection assay towards a rapid point-of-care test meeting the REASSURED criteria ${ }^{32}$, summarised in Supplementary Table 3. The incorporation of the amplification step on the LFA strip ${ }^{45}$ is a major challenge, along with sample processing and RNA extraction in resource-limited 
305 settings ${ }^{47}$, and removing the wash step. However, the sensitivity of this transduction 306 technique means there is leeway for sensitivity reductions, whilst retaining clinical 307 relevance: we have demonstrated single-copy detection with a 10min RT-RPA step, up to 30850 -fold greater sensitivity than the World Health Organisation viral suppression threshold of 3091,000 copies $/ \mathrm{mL}^{49}$. It is also flexible and easily translatable to other assays: amplification 310 methods using modified primers, including existing PCR assays, by changing only the 311 primers; direct detection by hybridisation of complementary modified probe sequences to a 312 molecular target; or protein detection in a sandwich assay using modified antibodies. In 313 order to demonstrate this, detection of the HIV-1 capsid protein using FNDs on paper was 314 evaluated, shown in Extended Data Figure 11, giving a LOD of 120fM. FNDs are also 315 applicable to a range of other in vitro diagnostic test formats. In addition, due to the long 316 fluorescence lifetimes of NV centres ${ }^{50}$ compared to nitrocellulose $^{7}$, time-gated fluorescence 317 measurements could be used to further improve FND-based LFA sensitivity.

318 The low power consumption (0.25W microwave power), optical readout, and 319 potential portability of this technique make it suitable for ultra-sensitive diagnosis and 320 monitoring in low-resource settings, with a portable fluorescence reader or smartphone321 based device including microwave modulation. The nature of lock-in readout makes it 322 robust to background light, minimising sensitivity losses when moving from a microscope to 323 such a portable device. FNDs on paper microfluidics offer a sensitive, robust labelling and 324 readout method for in vitro disease diagnostics.

\section{References}

326. Childress, L. \& Hanson, R. Diamond NV centers for quantum computing and quantum 327 networks. MRS Bulletin 38, 134-138 (2013).

328. Mochalin, V. N., Shenderova, O., Ho, D. \& Gogotsi, Y. The properties and applications of 329 nanodiamonds. Nature Nanotechnology 7, 11-23 (2012). 
33B. Boudou, J.-P. et al. High yield fabrication of fluorescent nanodiamonds. Nanotechnology 20, 331235602 (2009).

3324. Schirhagl, R., Chang, K., Loretz, M. \& Degen, C. L. Nitrogen-Vacancy Centers in Diamond:

333 Nanoscale Sensors for Physics and Biology. Annual Review of Physical Chemistry 65, 83-105 334 (2014).

335. Igarashi, R. et al. Real-time background-free selective imaging of fluorescent nanodiamonds 336 in vivo. Nano Letters 12, 5726-5732 (2012).

33\%. Leis, J., Martin, P. \& Buttsworth, D. Simplified digital lock-in amplifier algorithm. Electronics 338 Letters 48, 259 (2012).

3397. Shah, K. G. \& Yager, P. Wavelengths and Lifetimes of Paper Autofluorescence: A Simple 340 Substrate Screening Process to Enhance the Sensitivity of Fluorescence-Based Assays in 341 Paper. Analytical Chemistry 89, 12023-12029 (2017).

3428. Childress, L. et al. Coherent Dynamics of Coupled Electron and Nuclear Spin Qubits in 343 Diamond. Science 314, 281-285 (2006).

3449. Chang, H.-C., Hsiao, W. W.-W. \& Su, M.-C. Fluorescent Nanodiamonds (John Wiley \& Sons, 345 Ltd, Chichester, UK, 2018).

34610. Yu, S. J., Kang, M. W., Chang, H. C., Chen, K. M. \& Yu, Y. C. Bright fluorescent nanodiamonds: 347 No photobleaching and low cytotoxicity. Journal of the American Chemical Society 127, $348 \quad 17604-17605$ (2005).

34911. Shenderova, O. A. \& McGuire, G. E. Science and engineering of nanodiamond particle 350 surfaces for biological applications (Review). Biointerphases 10, 030802 (2015).

35112. Chang, Y. R. et al. Mass production and dynamic imaging of fluorescent nanodiamonds. 352 Nature Nanotechnology 3, 284-288 (2008). 
35313. Maze, J. R. et al. Nanoscale magnetic sensing with an individual electronic spin in diamond. $354 \quad$ Nature 455, 644-647 (2008).

35514. Balasubramanian, G. et al. Nanoscale imaging magnetometry with diamond spins under 356 ambient conditions. Nature 455, 648-651 (2008).

35715. Tetienne, J. P. et al. Magnetic-field-dependent photodynamics of single NV defects in 358 diamond: An application to qualitative all-optical magnetic imaging. New Journal of Physics 35914 (2012).

36016. Acosta, V. M. et al. Temperature Dependence of the Nitrogen-Vacancy Magnetic Resonance 361 in Diamond. Physical Review Letters 104, 070801 (2010).

3627. Hsiao, W. W. W., Hui, Y. Y., Tsai, P. C. \& Chang, H. C. Fluorescent Nanodiamond: A Versatile 363 Tool for Long-Term Cell Tracking, Super-Resolution Imaging, and Nanoscale Temperature 364 Sensing. Accounts of Chemical Research 49, 400-407 (2016).

36518. Vaijayanthimala, V. \& Chang, H.-C. Functionalized fluorescent nanodiamonds for biomedical 366 applications. Nanomedicine (London, England) 4, 47-55 (2009).

3679. Fu, C.-C. et al. Characterization and application of single fluorescent nanodiamonds as 368 cellular biomarkers. Proceedings of the National Academy of Sciences 104, 727-732 (2007).

3690. Chang, B. M. et al. Highly fluorescent nanodiamonds protein-functionalized for cell labeling 370 and targeting. Advanced Functional Materials 23, 5737-5745 (2013).

37D1. Waddington, D. E. et al. Nanodiamond-enhanced MRI via in situ hyperpolarization. Nature 372 Communications 8, 1-8 (2017).

37322. Hegyi, A. \& Yablonovitch, E. Molecular imaging by optically detected electron spin 374 resonance of nitrogen-vacancies in nanodiamonds. Nano Letters 13, 1173-1178 (2013). 
37523. Sarkar, S. K. et al. Wide-field in vivo background free imaging by selective magnetic 376 modulation of nanodiamond fluorescence. Biomedical Optics Express 5, 1190 (2014).

37724. Chapman, R. \& Plakhoitnik, T. Background-free imaging of luminescent nanodiamonds using 378 external magnetic field for contrast enhancement. Optics Letters 38, 1847 (2013).

3795. Doronina-Amitonova, L., Fedotov, I. \& Zheltikov, A. Ultrahigh-contrast imaging by 380 temporally modulated stimulated emission depletion. Optics Letters 40, 725 (2015).

38D6. Bhutta, Z. A., Sommerfeld, J., Lassi, Z. S., Salam, R. A. \& Das, J. K. Global burden, distribution, 382 and interventions for infectious diseases of poverty. Infectious Diseases of Poverty 3, 21 383 (2014).

3847. UNAIDS. Global HIV \& AIDS statistics - 2018 fact sheet. Tech. Rep. (2018).

3858. May, M. et al. Impact of late diagnosis and treatment on life expectancy in people with HIV386 1: UK Collaborative HIV Cohort (UK CHIC) Study. BMJ 343, d6016-d6016 (2011).

38729. Gray, E. R. et al. p24 revisited: a landscape review of antigen detection for early HIV 388 diagnosis. AIDS 32, 2089-2102 (2018).

38930. Price, C. P. Regular review: Point of care testing. BMJ 322, 1285-1288 (2001).

3901. World Health Organization. World Malaria Report. Tech. Rep. (2018).

39B2. Land, K. J., Boeras, D. I., Chen, X. S., Ramsay, A. R. \& Peeling, R. W. REASSURED diagnostics 392 to inform disease control strategies, strengthen health systems and improve patient 393 outcomes. Nature Microbiology 4, 46-54 (2019).

3983. Walter, J. G. et al. Protein microarrays: Reduced autofluorescence and improved LOD. 395 Engineering in Life Sciences 10, 103-108 (2010). 
3984. Kim, J. et al. Rapid and background-free detection of avian influenza virus in opaque sample 397 using NIR-to-NIR upconversion nanoparticle-based lateral flow immunoassay platform.

398 Biosensors and Bioelectronics 112, 209-215 (2018).

3995. Paterson, A. S. et al. A low-cost smartphone-based platform for highly sensitive point-of400 care testing with persistent luminescent phosphors. Lab Chip 17, 1051-1059 (2017).

40B6. Boudou, J. P., David, M. O., Joshi, V., Eidi, H. \& Curmi, P. A. Hyperbranched polyglycerol 402 modified fluorescent nanodiamond for biomedical research. Diamond and Related 403 Materials 38, 131-138 (2013).

4087. Hermanson, G. T. Zero-Length Crosslinkers. Bioconjugate Techniques 259-273 (2013).

40538. Gonzalez Flecha, F. L. \& Levi, V. Determination of the molecular size of BSA by fluorescence 406 anisotropy. Biochemistry and Molecular Biology Education 31, 319-322 (2003).

40739. Reth, M. Matching cellular dimensions with molecular sizes. Nature Immunology 14, 765408767 (2013).

40940. Ngom, B., Guo, Y., Wang, X. \& Bi, D. Development and application of lateral flow test strip 410 technology for detection of infectious agents and chemical contaminants: a review.

411 Analytical and Bioanalytical Chemistry 397, 1113-1135 (2010).

41211. Armbruster, D. A. \& Pry, T. Limit of blank, limit of detection and limit of quantitation. The 413 Clinical biochemist. Reviews / Australian Association of Clinical Biochemists 29 Suppl 1, 49$41452(2008)$.

41542. Daher, R. K., Stewart, G., Boissinot, M. \& Bergeron, M. G. Recombinase polymerase 416 amplification for diagnostic applications. Clinical Chemistry 62, 947-958 (2016).

41743. Lillis, L. et al. Cross-subtype detection of HIV-1 using reverse transcription and recombinase 418 polymerase amplification. Journal of Virological Methods 230, 28-35 (2016). 
41944. Crannell, Z. A., Rohrman, B. \& Richards-Kortum, R. Equipment-free incubation of 420 recombinase polymerase amplification reactions using body heat. PLOS ONE 9, 1-7 (2014).

42145. Rohrman, B. A. \& Richards-Kortum, R. R. A paper and plastic device for performing 422 recombinase polymerase amplification of HIV DNA. Lab on a Chip 12, 3082 (2012).

42346. Boyle, D. S., Lehman, D. A. \& Lillis, L. Rapid Detection of HIV-1 Proviral DNA for Early Infant 424 Diagnosis Using Rapid Detection of HIV-1 Proviral DNA for Early Infant Diagnosis. mBio 4, 425 00135-13 (2013).

42647. Dineva, M. A., Mahilum-Tapay, L. \& Lee, H. Sample preparation: a challenge in the 427 development of point-of-care nucleic acid-based assays for resource-limited settings. The 428 Analyst 132, 1193 (2007).

42948. Jauset-Rubio, M. et al. Ultrasensitive, rapid and inexpensive detection of DNA using paper 430 based lateral flow assay. Scientific Reports 6, 37732 (2016).

43149. Phillips, A. et al. Sustainable HIV treatment in Africa through viral-load-informed 432 differentiated care. Nature 528, S68-S76 (2015).

43350. Kuo, Y., Hsu, T.-Y., Wu, Y.-C., Hsu, J.-H. \& Chang, H.-C. Fluorescence lifetime imaging 434 microscopy of nanodiamonds in vivo. Advances in Photonics of Quantum Computing, 435 Memory, and Communication VI 8635, 863503 (2013).

\section{Acknowledgements}

437 We thank Matthew Schormans for help with circuit design, Michael Thomas for assistance 438 with dynamic light scattering measurements, and Martyn Towner for assistance with FTIR 439 measurements. This work was funded by the i-sense EPSRC IRC in Agile Early Warning 440 Sensing Systems in Infectious Diseases and Antimicrobial Resistance (EP/K031953/1 and 441 EP/R00529X/1); Royal Society Wolfson Research Merit Award to R.A.M. (WM130111); LCN 
442 Departmental Studentship to B.S.M; EPSRC Centre for Doctoral Training in Delivering 443 Quantum Technologies to G.D. (EP/L015242/1); H2020 European Research Council Local 444 quantum operations achieved through the motion of spins to J.J.L.M. (771493); and the 445 UCLH NHS Foundation Trust to J.H. and E.N.

446

447 448

\section{Author Contributions}

B.S.M., and R.A.M. conceived the research and led the study; P.J.D advised on nanodiamonds and J.J.L.M. on microwave modulation. B.S.M. showed the initial proof-of-concept; B.S.M. and L.B. designed and optimised the lock-in analysis, functionalisation and LFA design; B.S.M., L.B. and D.H. performed all the FND LFA experiments; H.D.G. designed, optimised and performed RT-RPA assays including primer design and template generation; D.H. adapted and performed RT-RPA assays and purification; J.J.L.M. and G.D. designed the microwave delivery including resonators; E.R.G. performed clinical RNA extraction, and advised on virology including primer design; J.H. performed qPCR on seroconversion panel; E.N. provided clinical expertise; B.S.M. and E.R.G. designed and performed binding site quantification experiments; B.S.M., L.B. and R.A.M. drafted the manuscript; and all authors reviewed and revised the manuscript.

\section{Competing Interests}

The authors declare the following competing financial interest(s): B.S.M., L.B., G.D., P.J.D., J.J.L.M. and R.A.M. are inventors on the UK patent application number 1814532.6.

\section{Correspondence}

Correspondence and requests for materials should be addressed to R. A. McKendry (email: r.a.mckendry@ucl.ac.uk).

\section{Methods}

Resonator design. CST Studio Suite 2015 (Dassault Systems) was used to create a 3D model of the resonator design, solving Maxwell's equations over a sweep of microwave 
frequencies to determine reflected and absorbed power. The design was based on copper patterned on a printed circuit board, using Rogers $4003 \mathrm{C}$ substrate for low dielectric loss at microwave frequencies. The top side had an interdigitated capacitor and a capacitorinductor omega-shaped loop, and the bottom had a ground plane. The dimensions of these components were varied iteratively to maximise the absorption at $2.87 \mathrm{GHz}$ and ensure an impedance of $50 \Omega$ for coupling to the frequency generator. The final design was exported as a 2D CAD file.

Preparation of functionalised FNDs. PG-functionalised FNDs were conjugated to Abs using DSC as shown in Extended Data Figure 3c. DSC activates hydroxyl surface groups to form succinimidyl carbonates, which can then react with antibodies to form stable carbamate or urethane bonds ${ }^{37}$.

In a typical synthesis, $100 \mu \mathrm{L}$ FND-PG $\left(1 \mathrm{mg} \mathrm{mL}^{-1}\right.$, Adamas Nanotechnologies, high brightness 120nm core + 20nm PG FND NDNV140nmHiPG2mg) were centrifuged at 21,130rcf for $7.5 \mathrm{~min}$ to condense the particles into a pellet. The supernatant was then removed and the FNDs were resuspended in anhydrous N,N-Dimethylformamide (DMF, 99.8\%, SigmaAldrich). After resuspension in DMF, the colloidal solution was sonicated for $5 \mathrm{~min}$ in an ultrasonic bath. The washing and sonication steps were repeated three times to remove water. After the last centrifugation, the particles were resupended in $100 \mu \mathrm{L}$ of a $50 \mathrm{mg} \mathrm{mL}^{-1}$ solution of DSC ( $\geq 95 \%$, Sigma-Aldrich) in DMF and placed in a thermoshaker for $3.5 \mathrm{~h}$ at $300 \mathrm{rpm}$ and room temperature. Excess reagents were removed by three cycles of centrifugation and resuspension in DMF (as described above). After the third centrifugation, the particles were resuspended in $100 \mu \mathrm{L}$ deionised water.

Depending on the desired surface functionalisation, $13.7 \mu \mathrm{L}$ of anti-digoxigenin antibodies (1 $\mathrm{mg} \mathrm{mL}^{-1}$, Abcam plc, ab76907) or $6.8 \mu \mathrm{L} \mathrm{BSA-biotin}\left(2 \mathrm{mg} \mathrm{mL}^{-1}\right.$ in deionised water, SigmaAldrich) were added to the activated FNDs. The mixture was placed in a thermoshaker overnight for $15 \mathrm{~h}$ at $300 \mathrm{rpm}$ and room temperature. The remaining succinimidyl carbonates were quenched by adding of $10 \mu \mathrm{L}$ of Tris- $\mathrm{HCl}$ pH $7.5(1 \mathrm{M}$, Thermo Fisher 
Scientific). After 30min, the unbound reagents were removed by three cycles of centrifugation and resuspension in deionised water $(100 \mu \mathrm{L})$ and stored in $100 \mu \mathrm{L}$ of PBS with $0.1 w t \%$ BSA.

After functionalisation, the FND concentrations were quantified by fluorescence intensity, as this remains unchanged during the functionalisation reactions: the fluorescence originates from the atomic structure of the FNDs, so is unaffected by surface modifications. This was carried out by performing a serial dilution of the FND stock solution (of know mass concentration, $c_{P}$, of $1 \mathrm{mg} \mathrm{mL}^{-1}$ based on manufacturers specifications) and using a spectrophotometer to measure the fluorescence compared to the functionalised-FND solution. A linear regression was fitted to the fluorescence intensity of the serial dilution of the stock FND solution against FND concentration and interpolated to calculate the mass concentration of the functionalised particles. Examples of this for the three different particle sizes are shown in Extended Data Figure 5c. This was converted to molar concentration $\left(C_{P}\right)$ using the diameter $(d)$, density of diamond $(\rho)$, and Avogadro constant $\left(N_{A}\right)$, shown in Methods Equation 1:

$$
C_{P}(\mathrm{M})=\frac{C_{P}\left(\mathrm{mg} \mathrm{mL}^{-1}\right)}{d^{3}\left(\mathrm{~nm}^{3}\right)} \cdot \frac{10^{3}}{\frac{\pi}{6} \cdot \rho\left(\mathrm{mg} \mathrm{nm}^{-3}\right) \cdot N_{A}\left(\mathrm{~mol}^{-1}\right)}
$$

Characterisation of Nanoparticles. Excitation spectra of the FNDs were acquired with a fluorescence microplate reader (SpectraMax i3, Molecular Devices LLC) and served as a reference to estimate the final FND concentration by comparison of the fluorescence intensity with the stock solution. Emission spectra were recorded with a spectrometer (SPM-002, Photon Control) with a 500nm LED light source (pE-4000, CoolLED). FTIR spectroscopy was performed by conjugating particles as described above, and storing in deionised water (maximum of 2 days), before centrifuging at 21,130rcf to condense the particles into a pellet and removing as much supernatant as possible to form a paste. This paste was pipetted onto the spectrometer (Bruker, Alpha). Three measurements of each sample were taken using 16 reads per measurement. Dynamic light scattering data and zeta 
potentials were measured with a Zetasizer (Zeta Sizer Nanoseries, Malvern Instruments Ltd) using a 150-fold dilution of the FNDs. The resulting number plots were fitted to the skewed exponential in Methods Equation 2 to find the peak diameter.

$$
N(x)=\frac{\exp \left(\frac{-(x-\mu)^{2}}{2 \sigma^{2}}\right) \operatorname{erfc}\left(\frac{-\alpha(x-\mu)}{\sqrt{2} \sigma}\right)}{\sqrt{2 \pi} \sigma}
$$

where $N$ is the number fraction, $x$ is the diameter, $\mu$ is the mean of the diameter distribution, $\sigma$ is the standard deviation, and $\alpha$ is the skew parameter.

Quantification of antibody binding sites on FND surface. In order to quantify the number of active antibody binding sites on the surface, an assay similar to PCR-ELISA and Kim et al. ${ }^{51}$ was developed. $300 \mu \mathrm{L}$ FNDs were functionalised with anti-DIG antibodies, as described in Methods, except the final suspension was in DNase/RNase-free distilled water (Thermo Fisher UltraPure) rather than storage buffer, and the particles were concentrated 5-fold (to $5 \mathrm{pM}, 60 \mu \mathrm{L})$. The suspension was subsequently split in half for a positive sample and a negative control, and $6 \mu \mathrm{L}$ of a $6 \mathrm{X}$ running buffer solution was added to each, to a final concentration of $1 \mathrm{X}$ running buffer ( $5 \%$ milk $+0.05 \%$ Empigen in water). A large excess of a DIG-modified DNA sequence $(0.9 \mu \mathrm{M}$ final concentration) was added to the positive sample, and the same excess of the same DNA sequence, but with no DIG modification was added to the negative control. A short DNA sequence (82bp) was used to avoid the bound DNA blocking available sites on the FND surface. The DIG-DNA was left to bind to the FND-Ab for $2 \mathrm{~h}$. After binding, each solution was diluted to $400 \mu \mathrm{L}$ in DNase/RNase-free distilled water before centrifuging at $376 \mathrm{rcf}$ for $2 \mathrm{~min}$ and removing the supernatant. This washing was repeated four times to remove excess DNA, with the final suspension in $150 \mu \mathrm{L} 100 \mu \mathrm{g} \mathrm{m}^{-1}$ salmon sperm DNA solution (Thermo Fisher UltraPure).

Quantitative PCR (qPCR) was then performed on the final suspensions. The template, primers and probe sequences are listed in Extended Data Figure 8d (assay taken from Besnier et $a .^{52}$ ). The master mix was the TaqMan Fast Virus 1-Step Master Mix (Thermo 
542 Fisher) with primers at $300 \mathrm{nM}$ and the probe at $150 \mathrm{nM}$, and $4 \mu \mathrm{L}$ of sample in a total

543 volume of $15 \mu \mathrm{L}$. The standard was constructed from serial dilutions of the pHRSIN-CSGW

544 plasmid $^{53}$. The qPCR was performed by an Applied Biosystems 7500 Real-Time PCR System

545 (Thermo Fisher), and the copy numbers quantified by the 7500 software (v2.0.6). The FND

546 concentrations in the final suspensions were measured as described in Methods. Dividing

547 the DNA copy number by the FND number gave the number of active binding sites per FND.

548 Target Amplification by Recombinase Polymerase Amplification. RNA template generation:

549 The template was designed using an alignment of 2929 clinical isolates of HIV-1 from the

550 Los Alamos HIV Sequence Database ${ }^{54}$ to identify conserved areas. The alignment was

551 mapped to the Dr Michael Edelstein using Geneious Software (version 10.0.6) and a highly

552 conserved region of 229 bp (1573-1801 bp from HXB2) selected to design five forward and

553 five reverse primers to be tested in RPA primer selection. Starting from a R9BALDEnv

554 plasmid (a gift from Greg Towers, UCL), DNA template was produced by polymerase chain

555 reaction amplification of the $1,503 \mathrm{bp}$ template sequence using the Phusion High-Fidelity

556 PCR Kit (New England Biolabs). Primer sequences used are shown in Extended Data Figure

$5578 \mathrm{~d}$. The thermocycling was performed at $98{ }^{\circ} \mathrm{C}$ for $30 \mathrm{~s}$, then 30 cycles of: $98{ }^{\circ} \mathrm{C}$ for $10 \mathrm{~s}, 65{ }^{\circ} \mathrm{C}$

558 for $20 \mathrm{~s}, 72{ }^{\circ} \mathrm{C}$ for $25 \mathrm{~s}$, and a final extension of $72{ }^{\circ} \mathrm{C}$ for $10 \mathrm{~min}$. The DNA was then

559 transcribed to RNA using the MEGAscript T7 Transcription Kit (Invitrogen) and purified using

560 MEGAclear Transcription Clean-Up Kit (Invitrogen), following the manufacturer's

561 instructions. The concentration of RNA template was measured via Qubit RNA HS assay kit

562 (Invitrogen) with the Qubit 4 Fluorometer.

563

564

565

566

567

568

569

$R T$-RPA reaction (amplicon serial dilution): RT-RPA assay was performed on a $1.5 \mathrm{~kb}$ HIV-1 in vitro transcribed RNA template. Optimisation of the assay is shown in Extended Data Figure 8. RT-RPA of the template was performed using TwistAmp Exo Reverse Transcription Kit (TwistDx), following the manufacturer's instructions. The reaction time was $30 \mathrm{~min}$ at $37^{\circ} \mathrm{C}$ shaking at 200rpm in an incubator (New Brunswick Innova 42). Nucleic acid sequences are listed in Extended Data Figure 8d, including a fluorescent probe. During amplification, exonuclease cuts the tetrahydrofuran, releasing the fluorescent tag (FAM) from the 
quencher, producing a quantitative signal. The resulting RPA products were incubated with RNAse A (QIAGEN) for $2 \mathrm{~h}$, before purification of amplified template to remove primers and fragments of RNA using QIAquick PCR Purification Kit (QIAGEN), following the manufacturer's instructions. Quantification by measuring absorption at $260 \mathrm{~nm}$ is confounded by RNA contamination, so dsDNA quantification was performed using a QuantiT PicoGreen dsDNA Assay Kit (Invitrogen), following the manufacturer's instructions. Fluorescence measurements were taken with a UV-visible spectrophotometer (Molecular Devices, SpectraMax i3).

$R T-R P A$ reaction (final assay with amplification): RT-RPA of the template was performed using TwistAmp Basic Kit (TwistDx). The master mix, containing 480nM of forward and reverse primers (for sequences see Extended Data Figure 8d, Integrated DNA Technologies), 1x rehydration buffer (TwistDx), reverse transcriptase (M-MLV Reverse Transcriptase, Invitrogen) and nuclease-free water (Invitrogen), was prepared in a tube. For each RPA reaction, $2 \mu \mathrm{L}$ of target HIV-1 RNA template was added to $45.5 \mu \mathrm{L}$ of master mix and a freeze-dried RPA pellet. The reaction was started by adding $2.5 \mu \mathrm{L}$ of magnesium acetate to each reaction, giving a final reaction volume of $50 \mu \mathrm{L}$. The RT-RPA reactions proceeded at 37 ${ }^{\circ} \mathrm{C}$ in a thermal incubator for $10 \mathrm{~min}$. The RT-RPA products were purified by QIAquick PCR Purification Kit (QIAGEN) and resuspended in a final volume of $50 \mu \mathrm{L}$ elution buffer for each reaction.

RT-RPA reaction (UCLH clinical standards): RNA from the UCLH HIV-1 viral load positive and negative standards (personal communication, gift from Paul Grant, UCLH) was extracted from a $140 \mu \mathrm{L}$ sample using the QIAamp Viral RNA Mini Kit (QIAGEN) essentially according to the manufacturer's instructions, except that elution was in $60 \mu \mathrm{L}$ water. $10 \mu \mathrm{L}$ of extracted RNA in water was used for each RT-RPA reaction.

RT-RPA reaction (seroconversion panel): RNA from an HIV-1 seroconversion panel (thirteen samples - ZeptoMetrix Corporation, Panel Donor No. 73698) was extracted from a $140 \mu \mathrm{L}$ sample using the QIAamp Viral RNA Mini Kit (QIAGEN) essentially according to the 
manufacturer's instructions, except that elution was in $60 \mu \mathrm{L}$ water. $2 \mu \mathrm{L}$ of extracted RNA in water was used for each RT-RPA reaction. The RT-RPA reactions proceeded at $37{ }^{\circ} \mathrm{C}$ in a thermal incubator for $10.5 \mathrm{~min}$. The RT-RPA products were purified by QIAquick PCR Purification Kit (QIAGEN) and resuspended in a final volume of $50 \mu \mathrm{L}$ elution buffer for each reaction.

Lateral flow assay. The following assays all use LFA strips with a poly-streptavidin test line, blocked by a proprietary polyvinylpyrrolidone-sucrose method (Mologic). The strips were $5 \mathrm{~mm}$ wide with the test line positioned $7 \mathrm{~mm}$ from the bottom of the strip.

A major challenge in developing sensitive LFAs is non-specific binding. To this end, sweeps of running buffers and washing buffers were performed to identify the combination giving the best SBR (see Extended Data Figure 6). This gave rise to a reduction in non-specific binding to the strip, reducing the blanks, and increasing the signal in turn. The optimum buffers in this study were found to be non-fat milk $5 w t \%+0.05$ vol\% Empigen in deionised water (running buffer) and 0.2 wt\% BSA with 0.2 vol\% Tween 20 in acetate buffer $10 \mathrm{mM} \mathrm{pH}$ 5 (washing buffer).

Having chosen running and washing buffers, the background was further reduced by optimising the concentration of FNDs, as shown in Extended Data Figure 7a-b. LFA strips were run with a dilution series of FND concentration. A positive test (500pM of DNA) and a negative control (deionised water) were run at each FND concentration. The fitted relationships between positive and negative lock-in amplitude signals and FND concentration were used, along with modelling of equilibrium binding, depending on antigen and FND concentration. This allowed the estimation of the LODs and dynamic ranges at each FND concentration, as explained in the Extended Data Figure 7c-d and Supplementary Information 2, leading to the selection of the FND concentration. The dynamic range is limited by the total number of FNDs at the top end and the non-specific signal in the negative at the bottom end. The chosen concentration gave a per-strip FND 
623 cost of less than $0.02 C$ ( $4.8 \mathrm{ng}$ of FNDs per strip). Total cost of consumables per test and estimated costs of a strip reader are shown in Supplementary Table 1.

625 The LFAs were performed by pipetting the solutions to be run into wells of a 96-well plate, 626 then dipping the strips into the wells. All LFAs were performed at room temperature.

627 Purified ssDNA concentrations were measured by absorption using the Nanodrop One ${ }^{\mathrm{C}}$ 628 (Thermo Scientific).

629

630

631

632

633

634

635

636

637

638

639

640

641

642

643

644

645

646

647

648

649

Assay with FND-BSA-biotin: BSA-biotin-functionalised FNDs were diluted in running buffer to the particle concentrations shown in Extended Data Figure $5 \mathrm{~d}$. $55 \mu \mathrm{L}$ of this suspension was run on each LFA strip.

Assay for model RT-RPA products: Initial optimisation and benchmarking was performed using a model ssDNA RT-RPA 'amplicon' (a short ssDNA strand with digoxigenin and biotin modifications at opposite ends), before moving to real RT-RPA amplicons for the final assay. A comparison of real RT-RPA amplicons with the model ssDNA 'amplicon' is shown in Extended Data Figure 9a, validating its use for optimisation, with similar $K_{D}$ values and dynamic ranges, although more variation in the blanks with real amplicons gives a higher LOD. A Monte Carlo simulation of the variances of the clinical sample lock-in amplitudes that can be explained by FND size distribution gives a value of $\sim 8-9 \%$ of the total variance (Extended Data Figure $9 \mathrm{~d}$ ). A further $\sim 0.1-2 \%$ of variance is explained by periodic drift in the modulation amplitude (Extended Data Figure 9e), and frequency noise contributes negligible variation (Extended Data Figure 9f), indicating that the majority is from other factors, such as strip-to-strip inconsistency. This strip-to-strip variation is more evident with larger FNDs, which could be because they are close to the minimum pore size of the nitrocellulose. LODs for the three FND diameters using the model ssDNA 'amplicon' is shown in Extended Data Figure 9b.

A single strand of DNA (26 bp), functionalised with digoxigenin at the 3 end and biotin at the 5 ' end (Integrated DNA Technologies, 5 ' biotin-GTCCGAGCGTACGACGAACGGTCGCTdigoxigenin 3') was used as a model for RT-RPA amplicons produced with biotin and 
digoxigenin functionalised primers. These model ssDNA strands were diluted in running buffer and $50 \mu \mathrm{L}$ of this solution was mixed with $5 \mu \mathrm{L}$ of anti-digoxigenin antibodyfunctionalised FND suspension (1,400, 170 and 3fM in PBS for 120, 200 and 600nm diameters, respectively). After $10 \mathrm{~min}$ at room temperature, these solutions were run on LFA strips. After all the solution was run (approximately $10 \mathrm{~min}$ ), the strips were transferred to wells of a 96 -well plate with $75 \mu \mathrm{L}$ of washing buffer ( $\sim 12 \mathrm{~min})$.

Assay for real RT-RPA products (amplicon serial dilution): After purification and quantification of amplicons, the assay was run and washed identically to the model RPA products above with FND concentrations of 2,600, 120 and 4fM for 120, 200 and 600nm diameters, respectively.

RT-RPA used a digoxigenin-modified forward primer and a biotin-modified reverse primer. The RT-RPA products, therefore, consist of dsDNA (181bp), each copy including a digoxigenin molecule at one end and a biotin molecule at the other. These modifications bind to anti-digoxigenin-functionalised FNDs, and the poly-streptavidin test line on the nitrocellulose paper, respectively, forming a sandwich structure and immobilising FNDs in the presence of amplicons, as shown in Figure $4 a$.

Final assay for RNA quantification with RT-RPA: After purification, $10 \mu \mathrm{L}$ of $6 \mathrm{X}$ running buffer (30wt\% non-fat milk with 0.3 vol\% Empigen in deionised water) was added to the $50 \mu \mathrm{L}$ RTRPA product. $5 \mu \mathrm{L}$ of anti-digoxigenin antibody-functionalised FND suspension was added before running the strips as above. For the lowest positive sample (average of 1.26 copies), there is a $71 \%$ chance of having at least one copy, based on the Poisson distribution. This gives a $26 \%$ chance of all four experimental replicates having at least one copy, using the binomial distribution, and a $42 \%$ chance for three of the four replicates. For the next dilution (average of 0.13 copies), these probabilities fall to $0.019 \%$ and $0.60 \%$. These probabilities are consistent with the results in Figure 4.

Fluorescence Modulation and Imaging. The paper strips were imaged using a fluorescence microscope (Olympus BX51) with a 550nm green LED as excitation light source (pE-4000, 
677 CoolLED), with a filter cube containing an excitation filter ( $500 \mathrm{~nm}$ bandpass, $49 \mathrm{~nm}$

678 bandwidth, Semrock), a dichroic mirror (596nm edge, Semrock) and emission filter (593nm

679 long-pass, Semrock). A 20x/0.4 BD objective (LMPlanFl, Olympus) was used. Images were 680 recorded with a high-speed camera (ORCA-Flash4.0 V3, Hamamatsu) using HCImage Live 681 software (Hamamatsu).

682 All strips were measured when dry to eliminate any possible variation due to drying during 683 measuring. Extended Data Figure 12 shows the detection on wet strips and the effect of 684 drying on the lock-in amplitude of the FND signal. This experiment was performed by 685 running positive and negative LFAs with the model 'amplicon' as above, then fixing each 686 strip to the microscope stage directly after completing the wash step. A 15-second lock-in 687 measurement at an exposure time of $20 \mathrm{~ms}$ was taken every $1 \mathrm{~min}$. The light source was only 688 on during measurement to prevent it speeding up drying. One of the negative controls was 689 measured for less than $55 \mathrm{~min}$ ( $35 \mathrm{~min}$ ), so its mean was used after this time in Extended 690 Data Figure 12. There is a small loss in sensitivity on wet strips ( 1.4-1.9x), corresponding 691 to a necessary increase in isothermal amplification time of less than $1 \mathrm{~min}$.

692 A microwave field was generated by a voltage controlled oscillator (VCO, Mini-Circuits, 693 ZX95-3360+) and a low noise amplifier (Mini-Circuits, ZX60-33LN+) connected to the 694 resonator circuit board (Minitron Ltd, Rogers 4003C $0.8 \mathrm{~mm}$ substrate and $1 \mathrm{ozft}^{-2} \mathrm{copper}$ 695 weight). The resonator was attached to the microscope stage. The tuning voltage of the 696 VCO was set to maximise the decrease in fluorescence. Modulation of the signal was 697 achieved by modulating the input voltage of the VCO with an on-chip reference frequency 698 generator at $4 \mathrm{~Hz}$ using a

$69932.768 \mathrm{kHz}$ crystal oscillator (DS32KHZ, Farnell Ltd) with a 14-stage frequency divider 700 (CD4060BM, Farnell Ltd). Circuit board design was performed using EAGLE (Autodesk). A 701 sweep of modulation frequencies was performed using this VCO and amplifier, using a 702 microcontroller (Arduino Nano 3.0) to generate the different modulation frequencies. 
703 The power dependence of the decrease in fluorescence was recorded using a benchtop 704 microwave generator (HM8135, Rohde \& Schwartz Hameg) and a low noise amplifier (Mini705 Circuits, ZRL-3500+). A broad sweep of microwave frequencies was measured with RF signal 706 generator (WindFreak Technologies LLC, SynthUSBII).

707 Computation lock-in and LOD. The fluorescence signal was modulated with a set modulation frequency $\left(F_{m}\right)$ and the amplitude of the resulting signal was computed with a computational lock-in algorithm. Images were recorded with the high-speed camera (ORCAFlash4.0 V3, Hamamatsu) at a sampling frequency $F_{s}$. Each frame was averaged to get a

711 mean pixel value at each time point $t_{0}=0$ to $t_{L}=L / F_{S}$, where $L$ was the total number of

712 frames. A moving average low-pass filter with a span width of $1.5 \cdot F_{s} / F_{m}$ was applied to the 713 fluorescence time series. The filtered signal, $V_{i n}$ was multiplied by two reference signals: in714 phase $\left(\sin \left(2 \pi F_{m} t\right)\right)$ and $\pi / 2$ out-of-phase $\left(\cos \left(2 \pi F_{m} t\right)\right)$ to obtain $V_{x}$ and $V_{y}$, respectively:

$$
\begin{aligned}
& V_{x}=V_{i n} \cdot \sin \left(2 \pi F_{m} t\right) \\
& V_{y}=V_{i n} \cdot \cos \left(2 \pi F_{m} t\right)
\end{aligned}
$$

715 The DC components of these two signals, $X$ and $Y$, were calculated by finding the mean of $V_{x}$ 716 and $V_{y}$, respectively, and enabled the evaluation of the magnitude $R$ of the lock-in 717 amplitude at the frequency $F_{m}$ according to:

$$
R=\sqrt{X^{2}+Y^{2}}
$$

718 Where there was no FND saturation (BSA-biotin assays), the LOD was computed by fitting 719 the lock-in amplitude, as a function of concentration, $c$, to a linear regression. Where there was saturation, (all assays except BSA-biotin assays) a Langmuir isotherm was fitted:

$$
S B R=k_{0}+k_{1} \cdot \frac{[\mathrm{T}]}{K_{D}+[\mathrm{T}]}
$$


721 where $k_{0}$ is SBR of the negative control, $k_{1}$ is a scaling constant representing the SBR at

722 target saturation, $[\mathrm{T}]$ in the amplicon concentration, and $K_{D}$ is the equilibrium dissociation

723 constant. Fitting was performed in Matlab using the fit $\mathrm{m}$ and nlinfit functions for linear and

724 Langmuir fits, respectively, weighting the fit by the variance at each concentration.

725 The LOD was defined as the intersection of the lower $95 \%$ confidence bound of the fit with

726 the upper $95 \%$ confidence bound of the blank measurements. ${ }^{41}$

\section{Methods References}

7281. Kim, E. Y. et al. A real-time PCR-based method for determining the surface coverage of 729 thiol-capped oligonucleotides bound onto gold nanoparticles. Nucleic Acids Research 34, 17307 (2006).

73152. Besnier, C., Takeuchi, Y. \& Towers, G. Restriction of lentivirus in monkeys. Proceedings of

732 the National Academy of Sciences of the United States of America 99, 11920-11925 (2002).

73353. Bainbridge, J. W. et al. In vivo gene transfer to the mouse eye using an HIV-based lentiviral 734 vector; efficient long-term transduction of cornealendothelium and retinal pigment 735 epithelium. Gene Therapy 8, 1665-1668 (2001).

7364. Foley, B. et al. HIV Sequence Compendium 2017 Editors. Eds. Published by Theoretical 737 Biology and Biophysics Group, Los Alamos National Laboratory, NM, LA-UR 18-25673 738 (2017).

7355. Kong, J. \& Yu, S. Fourier transform infrared spectroscopic analysis of protein secondary 740 structures. Acta Biochimica et Biophysica Sinica 39, 549-559 (2007).

74156. Zadeh, J. N. et al. NUPACK: Analysis and design of nucleic acid systems. Journal of 742 Computational Chemistry 32, 170-173 (2011). 
7437. SantaLucia, J. A unified view of polymer, dumbbell, and oligonucleotide DNA nearest-

744 neighbor thermodynamics. Proceedings of the National Academy of Sciences of the United

745 States of America 95, 1460-5 (1998).

7468. Laitinen, M. P. \& Vuento, M. Affinity immunosensor for milk progesterone: Identification of 747 critical parameters. Biosensors and Bioelectronics 11, 1207-1214 (1996).

\section{Data Availability}

749 The datasets generated during and/or analysed during the current study, and the computer 750 code used are available from the corresponding author on reasonable request, in line with 751 UCL and funder's requirements (EPSRC policy framework on research data).

\section{Extended Data Captions}

Extended Data Figure 1: Optimisation of microwave modulation. A linear resonator was designed to have a wideband response over the range $1-4 \mathrm{GHz}$, and an omega narrowband resonator was designed to have a stronger, narrower resonance at $2.87 \mathrm{GHz}$ with quality factor $Q=100$. The schematic printed circuit board layouts for the two resonators are shown in (a) and (d), respectively. The resulting simulated fields are shown in (b) and (e), respectively. The reflected power (S11) is plotted against frequency in (c) and (f). The narrowband resonator shows 5-6 orders of magnitude greater absorption than the wideband resonator at $2.87 \mathrm{GHz}$, indicating resonant coupling giving strong absorption. (f) Also shows the corresponding FND intensity dip. (g) Emission spectra of FNDs acted on by a $2.87 \mathrm{GHz}$ microwave field. The powers listed in decibel-milliwatts are the output power of the microwave generator (before the $17 \mathrm{~dB}$ amplifier). (h) Each spectrum is integrated over the whole wavelength range to give a total intensity, which is plotted against preamplifier power. This shows a linear relationship between fluorescence intensity and microwave power (in $\mathrm{dBm}$ ) above a threshold power, and up to $7 \mathrm{dBm}$, where the amplifier reaches its $1 \mathrm{~dB}$ compression power. At this point, the fluorescence starts to increase again due to a loss in the quality of the sinusoid leading to

768 power lost in harmonics. Error bars show the standard deviations, with 3 measurement repeats $\left(\mathrm{n}_{M}\right.$ $769=3)$.

771 Extended Data Figure 2: Optimisation of lock-in analysis. (a) Schematic of the computational lock-in

772 algorithm used to extract the microwave modulated FND signal from the background. The input 
signal is high-pass filtered using a moving average filter to remove low-frequency drift. It is

774 subsequently multiplied by cosine and sine functions with frequency $F_{m}$, and the resulting signals are

775 low-pass filtered to generate the in phase and quadrature components, respectively, of the vector 776 representation of the signal. The magnitude of this vector is calculated to remove the effect of 777 phase, giving the output magnitude. (b) The variation of lock-in amplitude with modulation rate $\left(F_{m}\right)$ 778 at various sampling rates $\left(F_{s}\right)$. A single strip with very high intensity was modulated at $F_{m} s$ between $7791-450 \mathrm{~Hz}$, and sampled at various $F_{s}$ s between $3.89-996 \mathrm{~Hz}$. The resulting plot shows that lock-in 780 amplitude is independent of $F_{s}$ when $F_{s}>2 F_{m}$. (c) and (d) show the relationships between lock-in 781 amplitude, exposure time $\left(T_{e}\right)$ and modulation frequency $\left(F_{m}\right)$. An identical LFA strip was measured 782 with exposure times between 10-50ms, using the maximum possible $F_{s}$ for each $T_{e}$, and $F_{m} S$ 783 between $1-15 \mathrm{~Hz}$. (d) shows $F_{m}$ against lock-in amplitude at various exposure times. It is shown that 784 the lock-in amplitude has its maximum $\sim 5 \mathrm{~Hz}$ for all frequencies, and reduces when $F_{m}$ is close to $F_{s} / 2$, its maximum possible value. This is evident in the raw signal plots in (c) for each $F_{m}$ at a fixed exposure time of $30 \mathrm{~ms}$. As $F_{m}$ approaches $F_{s} / 2$, the sampling effects obscure the square wave, decreasing lock-in amplitude. For maximum lock-in amplitude, the highest possible $T_{e}$ should be used. Here, we are limited to $50 \mathrm{~ms}$ by the background autofluorescence of the nitrocellulose, which saturates the camera above this value. A corresponding $F_{m}$ of $4 \mathrm{~Hz}$ was chosen as it is in the optimal range and is a power of 2 , so can be achieved by simply dividing the temperature compensation crystal oscillator (TCXO) frequency. (e) The variation of lock-in amplitude with total measurement time at $F_{m}=4 \mathrm{~Hz}$ and $F_{s}=20 \mathrm{~Hz}$ for five different concentrations of FNDs and a negative control, immobilised with a biotin-avidin interaction. The positive amplitudes stabilise quickly, reaching $5 \%$ of their $15 \mathrm{~s}$ value in $3.9 \mathrm{~s}$ for positive results. The negative results take longer to stabilise, reaching $5 \%$ of their $15 \mathrm{~s}$ value in 13s. A measurement time of $15 \mathrm{~s}$ (300frames) was used for subsequent 796 measurements. (f) Schematic circuit design of temperature compensation crystal oscillator (TCXO)based modulated microwave source. It is powered by a $5 \mathrm{~V}$ source which powers a TCXO, which outputs a $32.768 \mathrm{kHz}$ square wave. This is converted to a $4 \mathrm{~Hz}$ signal by a 4060 counter chip. This square wave controls two transistors which deliver $12 \mathrm{~V}$ stepped up power (DC converted) to the microwave VCO. The bias voltage is regulated from $12 \mathrm{~V}$ to $8.15 \mathrm{~V}$ by a voltage regulator. The VCO microwave output is amplified by the MW amplifier and transmitted to the omega resonator. (g) Printed circuit board layout of the prototype $(65 \mathrm{~mm} \times 38 \mathrm{~mm})$. Outputs for the microwave amplifier and microwave VCO are at the top right and bottom right, respectively. A photo of the printed circuit board with a pound coin for scale is shown below. 

nonspecific binding of various commercial FNDs with various surface functionalisations on LFAs. The lock-in amplitude at the test line was measured to quantify non-specific binding. The LFAs were also pre-blocked with a polyvinylpyrrolidone-sucrose solution (proprietary method, Mologic). The lowest non-specific binding was from the PG-functionalised particles (FND-PG), as the PG adds a hydrophilic layer. (b) Dynamic light scattering of three different FND particle core diameters: 120, 200 and $600 \mathrm{~nm}$. (c) A schematic of antibody functionalisation of FND-PG. Disuccinimidyl carbonate (DSC) activates hydroxyl surface groups to form succinimidyl carbonates, which can then react with antibodies to form stable carbamate or urethane bonds. (d)-(f) show scanning electron microscope images of FNDs with particle core diameters of 120, 200 and 600nm, respectively. (g) Dynamic light scattering was also used to measure the size and aggregated fraction after functionalisation of 120nm FND-PG before and after functionalisation with BSA-biotin or antibodies. Fitting the number plots to skew exponentials (Equation 3 in methods) gave peak particle hydrodynamic diameters of 106, 121 and 128nm. (h) The fitted peak diameters are plotted with error bars denoting their 95\% confidence intervals, showing no significant difference between the bio-functionalised diameters (FND-Biotin, FND-Ab), but both are significantly different from the pre-functionalisation diameter (FND-PG): * indicates a $p$-value of $\leq 0.05$ and ** a $p$-value of $\leq 0.01$ using a Tukey HSD post-hoc test. (i) FTIR spectroscopy of FND-PG and antibody-functionalised FND-PG. C-O and C-H peaks, indicative of the PG layer can be seen in both FND-PG and FND-PG-antibody at $\sim 1,100 \mathrm{~cm}^{-1}$ and $\sim 2,900 \mathrm{~cm}^{-1}$, respectively. The FND-PG-antibody spectrum displays additional peaks at $\sim 1,640 \mathrm{~cm}^{-1}$ and $\sim 1,540 \mathrm{~cm}^{-1}$, suggesting protein Amide I and Amide II bonds, respectively ${ }^{55}$, showing that protein functionalisation was successful.

Extended Data Figure 4: Quantification of the number of available binding sites per FND. (a) Initially, binding constants of the anti-digoxigenin (anti-DIG) antibody binding to DIG were measured using interferometry. Full experimental details are shown in Supplementary Information 1. Binding at different concentrations was measured and the resulting curves were fitted to exponentials. To find the equilibrium dissociation constant $\left(K_{D}\right)$, equilibrium binding values, $B$, were plotted here against concentration, $C$. A Langmuir adsorption isotherm was fitted $\left(B^{\infty}=\frac{a \cdot C}{K_{D}+C}\right)$ giving a $K_{D}$ value of $5.1 \times 10^{-10} \mathrm{M}$. (b) In order to find the on- and off-rates, $k_{\text {on }}$ and $k_{\text {off, }}$, the observed reaction rates, $k_{o b s}$, at each concentration were plotted and fitted to the linear relationship: $k_{o b s}=k_{o f f}+C \cdot k_{o n}$. The resulting fitted values are $k_{\text {on }}=1.6 \times 10^{5} \mathrm{M}^{-1} \mathrm{~s}^{-1}$ and $k_{\text {off }}=9.1 \times 10^{-5} \mathrm{~s}^{-1}$. (c) A schematic of the assay to quantify the number of available binding sites per FND. After functionalisation of FNDs with anti-DIG antibodies, a $\sim 50$-fold excess of DIG-modified DNA was added and left to bind for $2 \mathrm{~h}$. The negative 
DNA control used the same sequence, but with no DIG modification to compensate for non-specific binding and adequate washing. After multiple washes by centrifugation to remove the excess DNA, the remaining DNA (bound to FNDs) was quantified by qPCR. See Extended Data Figure 8d for template, primer and probe sequences, and Methods for full experimental details. (d) A kinetic binding simulation was performed to verify that all available sites would be occupied after $2 \mathrm{~h}$ with the above excess. The graph shows the fraction of sites on the FNDs which are occupied, with this $\sim 50$-fold excess, over a range of $K_{D}, k_{\text {on }}$ and $k_{\text {off }}$ values. The red cross in circle marks the location of the anti-DIG antibody used in this paper (using the values measured in (a) and (b)), indicating that $>99.9 \%$ of available sites will be occupied after $2 \mathrm{~h}$. This means that quantifying the DNA gives a true measure of available binding sites. (e) Amplification plot showing the normalised fluorescence intensity against number of cycles. A standard curve of each decade from 40 copies to $4 \times 10^{8}$ copies is plotted, along with the sample and negative control FND samples described above. The negative diluent controls are also plotted along with the $C_{q}$ threshold. The shaded areas show the standard deviation of repeats ( $\mathrm{n}_{T}=3$ for standard curve and $\mathrm{n}_{T}=6$ for samples). (f) The resulting $C_{q}$ values are plotted against copy number per reaction. Error bars show standard deviations $\left(\mathrm{n}_{T}=3\right.$ for standard curve and $\mathrm{n}_{T}=6$ for samples). The standard curve was fitted to a logarithmic curve $\left(C_{q}=-3.2 \log _{10}\right.$ copies + 39), allowing calculation of the number of copies in the DIG-DNA sample and negative DNA control. Dividing by the particle concentration (measured as shown in Extended Data Figure $5 \mathrm{c}$ ) and subtracting the negative DNA control value, gives the number of available binding sites per particle as 4,300 sites. This is within what is geometrically plausible, giving an area per antibody of at least $200 \mathrm{~nm}^{2}$ (assuming at least 1 paratope available of at least $75 \%$ of the bound antibodies). The corresponding calculated values for 120 and 200nm particles are 172 and 477 available binding sites per FND respectively, assuming the same loading density.

Extended Data Figure 5: Lateral flow and FND benchmarking. (a) Measurement of flow rate of lateral flow strips. During wetting, the flow follows the Washburn equation, where $V \sim t^{\frac{1}{2}}$ (inset), and during fully-wetted flow, Darcy's law for capillary flow is followed $(V \sim t)$, with a constant flow rate of $6.9 \mu \mathrm{Lmin}^{-1}$. (b) Using a one-to-one receptor-ligand binding approximation, the binding of biotinylated FNDs to streptavidin was modelled kinetically, indicating that all the FNDs bind with a residency time of $>\sim 10^{-3} \mathrm{~s}$. Here, the residency time is measured as $4 \mathrm{~s}$, using the flow rate from (a), so all the FNDs should bind. (c) An example of the measurement of FND concentration. FND fluorescence is unaffected by surface chemistry, so is used to quantify concentration. A serial dilution of FND suspensions was performed, from a known stock concentration (filled circles with error bars showing standard deviations). This was then fitted with a linear regression (lines) to find a 
relationship between fluorescence intensity and concentration. After each FND functionalisation, the final suspensions' fluorescence intensities were measured, and the linear fit was used to estimate concentration (crosses). (d) Fundamental LODs for different size FNDs on LFAs, using a model biotin-avidin interaction. $55 \mu \mathrm{L}$ suspensions of BSA-biotin-functionalised FNDs were run at different concentrations on poly-streptavidin strips. Concentrations were chosen to span the dynamic range of the camera, limited by over-exposure, as seen with the top concentration of 200 and 600nm FNDs. Error bars show standard deviations $\left(n_{T}=3, n_{M}=3\right)$. Each series is fitted to a simple linear regression, shown as the solid line, with 95\% confidence intervals shown shaded. LODs for 120, 200 and 600nm diameter FNDs are 200aM, 46aM, and 820zM respectively, defined by the intersection of the lower $95 \%$ confidence intervals of the linear fit with the upper $95 \%$ confidence intervals of the blanks for each particle size.

Extended Data Figure 6: Assay optimisation by buffer selection. Sensitivity is limited by the nonspecific binding of FNDs at the LFA test line. LFA strip blocking, running buffer and washing step are, therefore, key factors in improving LOD. In this section 120nm FNDs were used for optimisation. (a) Signal-to-background comparison for the in different running buffers. There is no wash step. Error bars show standard deviations $\left(\mathrm{n}_{M}=3\right)$. Milk was selected as the basis for the running buffer. (b) Subsequently, a sweep of different surfactants was performed $\left(\mathrm{n}_{M}=1\right)$. The best signal-tobackground ratio came from adding $0.05 \mathrm{vol} \%$ Empigen, showing a significant increase in the signalto-background. There is no wash step. (c) The best running buffer was then used for a washing buffer $\mathrm{pH}$ sweep $\left(\mathrm{n}_{M}=1\right)$. All washing buffers were run at a volume of $75 \mu \mathrm{L}$, chosen because preliminary experiments showed it to be a good compromise between assay time and washing success. Although results were similar, $\mathrm{pH} 5$ gave the best signal-to-background ratio, so acetate buffer at $10 \mathrm{mM} \mathrm{pH} 5$ was used as the basis for a second washing buffer sweep, shown in (d), testing a number of detergents and adding casein at $0.2 \mathrm{wt} \%$ as a blocking protein $\left(\mathrm{n}_{M}=1\right)$. As a final test, the three best running buffers were tested, each with the three best washing conditions, displayed as a grid in (e). Each square is the average of three measurements $\left(n_{M}=3\right)$. The results were consistent with previous sweeps, the combination of the best running buffer and best washing buffer giving the best signal-to-background. Milk and protein percentages are by weight and detergent percentages are by volume.

Extended Data Figure 7: Optimisation of FND concentration. The background was reduced by optimising the particle concentration, shown here for 120nm FNDs. (a) A positive LFA strip (500pM of ssDNA) and a negative control (deionised water) were run at varying FND concentrations 
between 3.88fM and 496fM, plotted against FND concentration, and fitted to simple linear regressions. The error bars show the standard deviations of repeat measurements $\left(n_{M}=3\right)$. Linear

910 regressions are shown by solid lines, and shaded areas show the $95 \%$ confidence intervals of the fits.

911 (b) Signal-to-background ratio, found by dividing the fitted linear regressions in (a), is plotted against

912 FND concentration. At higher concentrations, where the gradient term of the linear regression

913 dominates, the positive and negative lock-in values tend to a constant separation on the log-log

914 plot, so the signal-to-background ratio tends to a constant value of $\sim 27$. At low concentrations, the

915 positive and negative curves converge as the negative lock-in amplitude levels off at the noise

916 threshold, and the signal-to-background ratio tends to 1. (c) The fitted linear regressions in part (a)

917 were used, along with the antibody equilibrium dissociation constant measured in Extended Data

918 Figure 4, to estimate the variation of lock-in amplitude with analyte concentration at different FND

919 concentrations. The principles and equations are described in full in Supplementary Information 2.

920 The LOD for each FND concentration is defined as the intersection of this plot with the value of the

921 blank plus two times the 95\% confidence interval at that value, assuming a low concentration

922 positive would have a similar confidence interval. (d) The estimated LODs and dynamic ranges from

923 (c), plotted against FND concentration, to determine the optimum.

925 Extended Data Figure 8: Primer Optimisation. (a) List of forward primers (F1-F5) and reverse primers (R1-R5) tested for the initial primer screen. (b) An initial primer screen was performed to achieve the highest amplification efficiency $\left(n_{T}=3\right)$ using the TwistAmp Exo Reverse Transcription Kit (TwistDx). The yield of each primer combination was measured by the fluorescence of the exo probe with a fluorescence microplate reader (SpectraMax i3, Molecular Devices LLC). Primers F5 and R3 gave the highest yield, although all the yields were above $63 \%$ of this value. (c) Interactions between forward primers and reverse primers to predict the minimum free energy structures for the ten primer combinations that gave the largest yield of RPA product in the primer screen. The table shows the results of simulations in NUPACK ${ }^{56}$, using an input of $10 \mu \mathrm{M}$ for each oligonucleotide. The minimum free energy secondary structures are the most energetically favourable secondary structures that can be assumed for oligonucleotides of a given primary sequence, calculated using the nearest-neighbour method ${ }^{57}$. Primers F1 and R4 were selected for future work since the energetics of their hybridisation are much less favourable than that of F3 and R5, yet they still gave a high RPA yield in the primer screen (93\% of the highest yield pair). (d) A list of oligonucleotides used for PCR, RPA and qPCR assays. The PCR reverse primer included a T7 promoter for RNA transcription (underlined) and a spacer (bold). (e) Gel electrophoresis of 1,503bp template sequence 
produced by PCR using a 1\% agarose gel. (f) Gel electrophoresis of 181bp double-stranded RT-RPA

942 products using a $1 \%$ agarose gel.

Extended Data Figure 9: Comparison of LODs of model ssDNA with real RPA amplicons and gold nanoparticles. (a) The dilution series of the real RPA amplicons and the model ssDNA 'amplicons' were plotted against concentration for 600nm FNDs (dots with error bars showing standard deviations, $\mathrm{n}_{T}=3-9, \mathrm{n}_{M}=3$ ) with their respective linear fits (solid lines with $95 \%$ confidence intervals of the fit shown shaded). The curves are similar, with fitted $K_{D}$ values of 29 and $22 \mathrm{fM}$ for model and real amplicons, respectively, and similar dynamic ranges. The real amplicons showed increased variation in the blanks, leading to a higher blank cutoff giving a higher LOD, and slightly reduced signal-to-blank ratio. (b) The dilution series of model ssDNA 'amplicons' were plotted against concentration for 120, 200 and 600nm FNDs (dots with error bars showing standard deviations, $\mathrm{n}_{T}=$ $3, n_{M}=3$ ) with their respective linear fits (solid lines with $95 \%$ confidence intervals of the fit shown shaded). The LODs are 3.7, 3.6 and $0.8 \mathrm{fM}$ respectively. (c) Comparison of 600nm FNDs with 40nm gold nanoparticles on LFAs, commonly used in LFAs due to a good compromise between stability (and therefore ease of functionalisation), and sensitivity ${ }^{58}$. Serial dilutions are plotted (dots with error bars showing standard deviations, $\mathrm{n}_{T}=3, \mathrm{n}_{M}=3$ for the FNDs; and dots with error bars showing the standard deviations across the test line, $\mathrm{n}_{T}=1, \mathrm{n}_{M}=1$ for the gold nanoparticles) LODs are calculated as previously, giving $800 \mathrm{aM}$ and 6.0pM, respectively. (d) A Monte Carlo simulation of the signal variation that can be explained by the FND size distribution (from DLS measurements in Extended Data Figure $3 b)$ was performed $(n=200,000)$. The violin plots show the normalised simulated random variation in lock-in amplitudes due to the $600 \mathrm{~nm}$ FND size distribution in the clinical sample assays in Figure $4 \mathrm{~d}$ (negative plasma control and clinical standard). The experimental data is overlaid, showing that FND size distribution explains $\sim 8-9 \%$ of the total experimental signal variance. A further $\sim 0.1-2 \%$ of the variance is explained by periodic drift in modulation amplitude, shown over $45 \mathrm{~min}$ in (e), normalised to the mean. (f) shows a plot of the variation in lock-in amplitude due to small changes in the modulation frequency, $F_{m}$. The variance of the frequency is 3 $\times 10^{-8} \%$ over the same period, giving negligible differences in lock-in amplitude. Full details of the simulation are given in Supplementary Information 3.

Extended Data Figure 10: Further analysis of RT-RPA samples. (a) ANOVA analysis was performed on the measured lock-in amplitudes of the FND LFAs, giving a $P$ value of $7.4 \times 10^{-29}$ and $F$ value of concentration). The horizontal red lines represent the medians, the horizontal blue lines represent 
the 25th and 75th percentiles and the notches represent the 95\% confidence intervals of the medians. The black dashed lines represent the range for each group. (b) A graphical comparison of the means of the groups (grouped by RNA concentration). The circles represent the means, and the horizontal lines represent the comparison intervals of the means (overlap of these intervals denotes statistical similarity). The negative control, highlighted in blue, is shown to be not significantly different from the $10^{-2}$ and $10^{-1}$ RNA copy number samples ( $P$ values $>0.999$, shown in grey), but it is significantly different from the $1,10^{1}$, and $10^{2}$ RNA copy number samples ( $P$ values $\sim 10^{-8}$, shown in red). (c) A table of ANOVA $P$ values. The $P$ value for the null hypothesis that the difference between the means of the two groups is zero. (d) Comparison of amplification time for a low copy number RT-RPA sample (average of 1.26 RNA copies). Multiple RPA reactions were run and stopped after different times, before adding to FND LFAs, as described in methods. A negative control is shown for comparison, and the dashed line represents the upper 95\% confidence interval of the negative control. Dots show the mean of the measurement repeats $\left(n_{M}=3\right)$, crosses show the individual measurements, and error bars represent the standard deviation. (e) Early disease detection using FND LFAs was demonstrated by a seroconversion panel (ZeptoMetrix Corporation, Panel Donor No. 73698), taken from a single donor over a period of six weeks spanning the early stages of an HIV-1 infection. The thirteen samples of the panel were measured on FND LFAs $\left(\mathrm{n}_{E}=1-2, \mathrm{n}_{M}=3\right)$. The measured values are plotted along with positive and negative non-amplification controls. They are colour-coded for RT-PCR results, and labelled with sample numbers, dates, and copy numbers in brackets. The blank cutoff is defined as the upper $95 \%$ interval of the negative control. The results show that the RNA was detectable on FND LFAs as early as RT-PCR, and 6/7 RT-PCR-positive samples were detected on FND LFAs, whilst 6/6 RT-PCR-negative samples were negative.

Extended Data Figure 11: Detection of HIV-1 capsid protein on using 600nm FNDs. A serial dilution of the capsid protein was detected on streptavidin-modified LFAs using a sandwich of a biotinylated capture nanobody and antibody-modified FNDs. The results are plotted $\left(n_{E}=3-4, n_{M}=3\right)$, normalised to the blanks for each sample set, and fitted to a Langmuir curve (Methods Equation 6). This gives a LOD of $120 \mathrm{fM}$, and a lowest concentration that is significantly different from the blank (at the $95 \%$ confidence level) of $3 \mathrm{pM}$, marked with *. Full experimental details are shown in Supplementary Information 4.

\section{Extended Data Figure 12: Effect of lateral flow test strip drying on lock-in amplitude of FND assay.}

(a) Positive and negative lateral flow test strips were measured over time after completed running (time $=0$ ), showing a small increase in the positive strip lock-in amplitude as the strip dries (the 
1009 initial lock-in amplitude is $\sim 70 \%$ of the final value), however no increase is seen in the negative 1010 control. The shaded areas show the standard deviation between repeats $\left(n_{T}=3\right)$. (b) The resulting 1011 signal-to-blank ratio variation over time. The shaded areas show the standard deviation between 1012 repeats $\left(n_{T}=3\right)$, showing that the effect of drying is quite small compared to strip-to-strip variation. 
NV-Center

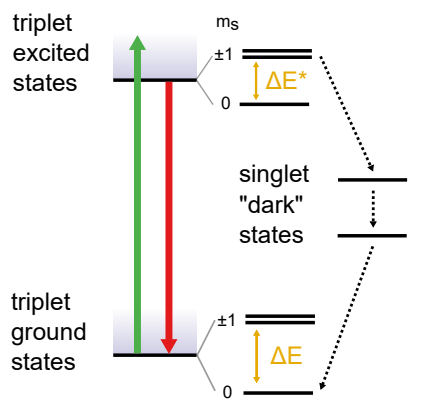

b

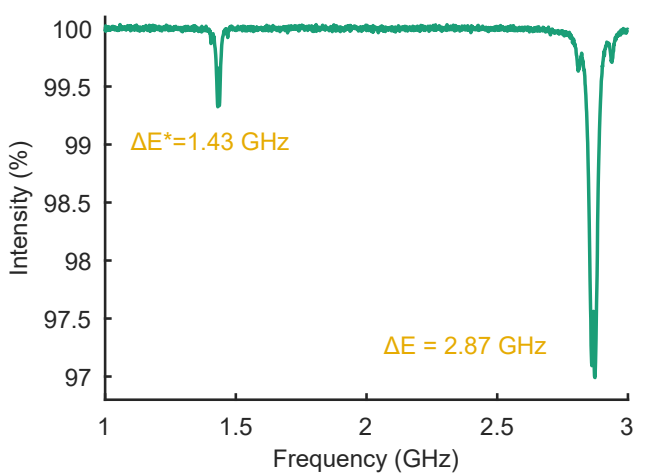

e d

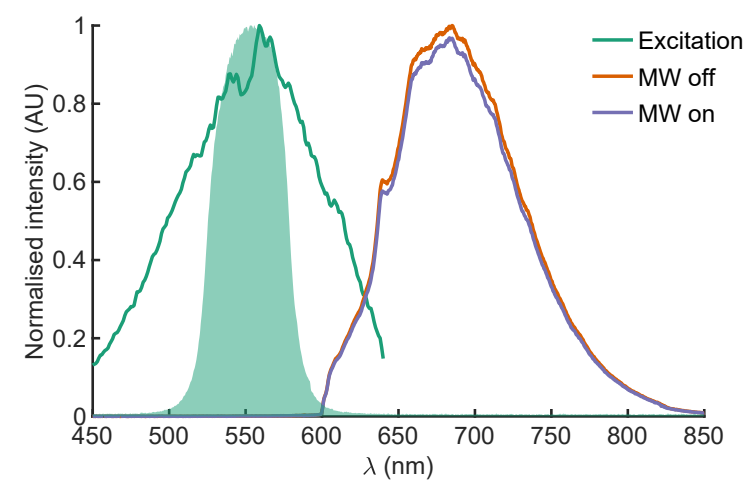

c
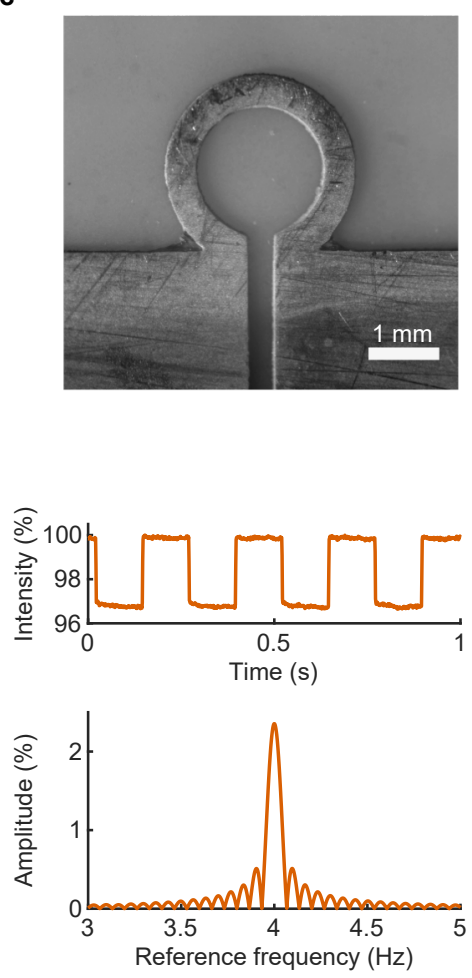
FND-BSA-Biotin

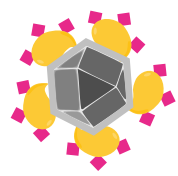

Streptavidin

Paper

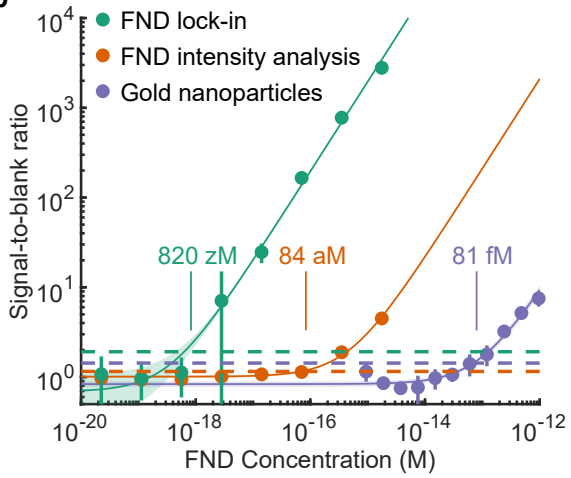

FND Concentration
$1.8 \mathrm{fM}$
$70 \mathrm{aM}$
$2.8 \mathrm{aM}$
$110 \mathrm{zM}$
$0 \mathrm{M}$

Fluorescence images

Signal modulation (pixel variation)

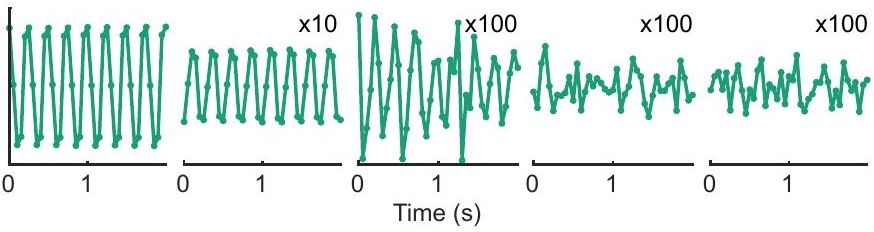


